\title{
Variability in word-recognition performance
}

\author{
CHRISTINE BROWNING-CRINION, ROBERT DOLMETSCH, and M. S. MAYZNER \\ Loyola University, Chicago, Illinois 60626
}

The present study, employing a backward visual masking paradigm and a computer-based CRT display system, examines the word-recognition performance for 220 four-, five-, and sixletter words, with a sample of 50 subjects. Results, as found previously, continue to demonstrate very large individual variabilities in such information processing tasks.

For about the past 10 years we have been concerned with developing a better understanding of pattern recognition in general, and letter and word recognition in particular (Mayzner, 1972, 1975a, 1975b; Mayzner \& Greenberg, 1971; Mayzner, Tresselt, \& Helfer, 1967). A persistent and puzzling result in almost all of the above-mentioned letter- and word-recognition studies has been the very significant variability found between subjects in letter- and word-recognition performance. Typically, we have found, for groups of college subjects selected at random, that, almost independently of stimulus parameters, individual differences between subjects for the identical stimulus items can and do vary from a few percent correct recognitions to almost $100 \%$ correct recognitions, in a variety of backward visual masking paradigms (Mayzner, 1972, 1975a, 1975b; Mayzner \& Greenberg, 1971).

As a result of these large individual differences, it seemed reasonable to ask: "If we employed a large number of words [e.g., 220] and a large number of subjects [e.g., $\mathrm{N}=50$ ], to what extent should we continue to find such differences?" The present study directly confronted this question.

\section{METHOD}

\section{Subjects}

Fifty volunteer subjects were employed, 29 females and 21 males, ranging in age from 17 to 56 years.

\section{Procedure}

A total of 220 words, four, five, or six letters in length and selected with respect to three frequency groupings from the Kucera and Francis (1967) word list, were employed as stimuli, as shown in Table 1. A computer-based cathode-ray tube (CRT) display system, described previously (Mayzner, 1975a), was used to present the 220 word stimuli and involved a PDP-8/E digital computer driving a VR-14 CRT display coated with a P24 phospher. Each subject was seated $2 \mathrm{ft}$ in front of the CRT display, with the head positioned firmly in a head-and-chin rest. Each letter of the four-, five-, or six-letter words was approximately 1 -in. high and $3 / 4$-in. wide. Each of the 220 words, displayed in a different random order to each subject, was presented for a fixed duration of $40 \mathrm{msec}$, followed immediately by a 500 -msec noise field or mask, as described earlier (Mayzner,

This research was supported in part by NSF Grant BNS 75-09800 A02 to M. S. Mayzner.
1972, 1975a, 1975b). After each presentation or display (i.e., a word followed immediately by a noise field or mask), the subject was requested to write all the letters that had been perceived or the entire word, if the entire word had been recognized correctly in its entirety by each subject.

\section{RESULTS AND DISCUSSION}

Table 1 gives all the words employed in the study, broken down by word length and word frequency, and also presents the percent correct recognitions for each of the 220 words examined, based on the 50 subjects studied. As may be easily seen in Table 1, there are

Table 1

All Words Employed in the Study, Shown by Word Length and Frequency Count and Giving Percent Correct Word Recognitions

\begin{tabular}{cccccc}
\hline Four & Percent & Five & Percent & Six & Percent \\
Letters & Correct & Letters & Correct & Letters & Correct \\
\hline
\end{tabular}

Kučera and Francis Word Frequency: 1 Per Million

$\begin{array}{lrlrlr}\text { bred } & 30 & \text { amity } & 6 & \text { asylum } & 42 \\ \text { clod } & 34 & \text { avert } & 16 & \text { bigots } & 16 \\ \text { faze } & 6 & \text { basil } & 28 & \text { cavort } & 16 \\ \text { hash } & 46 & \text { chaps } & 32 & \text { creamy } & 36 \\ \text { luca } & 18 & \text { crust } & 44 & \text { embryo } & 34 \\ \text { jade } & 40 & \text { exalt } & 42 & \text { fiesta } & 18 \\ \text { keno } & 16 & \text { flirt } & 48 & \text { kneels } & 36 \\ \text { lurk } & 50 & \text { graze } & 12 & \text { lewdly } & 10 \\ \text { moth } & 44 & \text { jerks } & 42 & \text { ouster } & 10 \\ \text { oops } & 50 & \text { joked } & 40 & \text { parcel } & 40 \\ \text { prim } & 16 & \text { kiosk } & 4 & \text { purses } & 30 \\ \text { puff } & 38 & \text { liars } & 24 & \text { quirks } & 18 \\ \text { quam } & 2 & \text { mince } & 14 & \text { reaped } & 10 \\ \text { rims } & 30 & \text { omega } & 24 & \text { ribald } & 10 \\ \text { runt } & 40 & \text { plasm } & 14 & \text { stigma } & 26 \\ \text { teas } & 22 & \text { query } & 16 & \text { suntan } & 46 \\ \text { whoa } & 16 & \text { rogue } & 28 & \text { topple } & 46 \\ \text { yolk } & 36 & \text { spoof } & 38 & \text { tycoon } & 52 \\ & & \text { tidal } & 22 & \text { uplift } & 22 \\ & & \text { twigs } & 18 & \text { vocals } & 4 \\ & & \text { usurp } & 16 & \text { wilted } & 24 \\ & & \text { volts } & 10 & \text { yelped } & 34 \\ & & \text { zoned } & 22 & \text { zombie } & 4\end{array}$

Kučera and Francis Word Frequency: 15 to 25 Per Million

$\begin{array}{lllrll}\text { bees } & 44 & \text { alien } & 22 & \text { beaten } & 42 \\ \text { burn } & 56 & \text { alter } & 28 & \text { buffer } & 38 \\ \text { clue } & 44 & \text { blast } & 48 & \text { devote } & 36 \\ \text { damp } & 26 & \text { boost } & 4 & \text { ethics } & 50 \\ \text { drag } & 20 & \text { cubic } & 44 & \text { fabric } & 38 \\ \text { earn } & 52 & \text { deals } & 34 & \text { gotten } & 24\end{array}$


Table 1 Continued

\begin{tabular}{cclclc}
\hline $\begin{array}{c}\text { Four } \\
\text { Letters }\end{array}$ & $\begin{array}{c}\text { Percent } \\
\text { Correct }\end{array}$ & $\begin{array}{c}\text { Five } \\
\text { Letters }\end{array}$ & $\begin{array}{c}\text { Percent } \\
\text { Correct }\end{array}$ & $\begin{array}{c}\text { Six } \\
\text { Letters }\end{array}$ & $\begin{array}{r}\text { Percent } \\
\text { Correct }\end{array}$ \\
\hline flag & 28 & dutch & 54 & helium & 42 \\
grab & 22 & elder & 38 & ideals & 40 \\
hers & 44 & fancy & 48 & judged & 46 \\
jeep & 32 & farms & 18 & kissed & 54 \\
kick & 54 & gates & 20 & lyrics & 42 \\
lawn & 28 & ivory & 40 & madame & 16 \\
monk & 30 & knock & 56 & needle & 54 \\
nest & 60 & loses & 38 & occupy & 4 \\
oils & 38 & lover & 10 & oppose & 36 \\
pill & 48 & marry & 12 & picnic & 42 \\
quit & 26 & nurse & 60 & potato & 42 \\
rope & 42 & onion & 50 & rental & 46 \\
spun & 46 & pains & 20 & rubber & 58 \\
swim & 20 & razor & 28 & tennis & 60 \\
ties & 54 & sheer & 50 & vessel & 10 \\
unto & 20 & stove & 50 & wasted & 30 \\
zero & 26 & votes & 28 & whites & 28 \\
& & waved & 14 & yankee & 52
\end{tabular}

Kučera and Francis Word Frequency: 100 to 400 Per Million

\begin{tabular}{lllllr} 
able & 32 & among & 18 & across & 14 \\
area & 36 & areas & 10 & behind & 52 \\
arms & 18 & black & 50 & center & 60 \\
book & 60 & board & 28 & charge & 2 \\
case & 36 & books & 52 & common & 40 \\
cost & 38 & child & 56 & effect & 42 \\
find & 60 & class & 50 & either & 48 \\
full & 56 & clear & 58 & figure & 58 \\
girl & 42 & death & 56 & future & 64 \\
hard & 56 & early & 50 & having & 10 \\
keep & 58 & field & 26 & itself & 36 \\
knew & 10 & force & 54 & looked & 48 \\
land & 64 & front & 54 & moment & 46 \\
love & 24 & going & 34 & mother & 48 \\
miss & 30 & group & 56 & office & 50 \\
name & 54 & hands & 40 & others & 60 \\
plan & 40 & hardy & 52 & period & 66 \\
play & 58 & later & 50 & policy & 50 \\
pool & 70 & level & 50 & reason & 36 \\
rate & 46 & music & 40 & result & 50 \\
says & 46 & north & 64 & second & 56 \\
seem & 34 & quite & 42 & seemed & 38 \\
town & 38 & short & 62 & social & 42 \\
true & 66 & sound & 58 & street & 68 \\
type & 62 & stood & 52 & toward & 28 \\
west & 42 & total & 50 & wanted & 42 \\
wife & 34 & voice & 44 & within & 34 \\
york & 46 & women & 32 & & \\
& & wrong & 42 & & \\
& & young & 58 & & \\
\hline
\end{tabular}

considerable individual differences in word-recognition scores. More importantly, Table 2 presents the individual recognition scores, ordered from smallest to largest, for each of the 50 subjects in the study. As has been found before (Mayzner, 1972, 1975a, 1975b; Mayzner \& Greenberg, 1971), the variability in word-recognition performance between subjects is very large and appears to be a very stable and reproduceable finding. For example, Table 2 shows that for this study, in which 220 different words were shown to 50 different subjects, 10 subjects failed to recognize any words whatsoever, while the recognition scores for the remaining 40 sub- jects ranged from a low of 12 correct recognitions to a high of 185 correct recognitions, out of a total maximum correct recognition score of 220 . While, clearly, no attempt will be made here to provide an explanation for these very large individual differences, their empirical reality must ultimately be accounted for in any comprehensive visual information processing model of word-recognition processes.

While not a major objective of this study, word length and word frequency also varied and the percent correct recognitions for these variations are presented in Table 3.

As shown in Table 3, increases in word frequency led to significant increases in word-recognition scores, that is, from $26.47 \%$ correct recognitions $(p<.001)$ to $44.37 \%$ correct recognitions, as expected, while increases in word length surprisingly led to very small and completely insignificant decreases in word-recognition scores, that is, from $37.31 \%$ correct recognitions to $36.05 \%$ correct recog-

Table 2

A Listing of All 50 Subjects and Their Associated Recognition Scores (Maximum = 220)

\begin{tabular}{|c|c|c|c|}
\hline Subjects & N Correct & Subjects & N Correct \\
\hline 1 & 0 & 26 & 81 \\
\hline 2 & 0 & 27 & 86 \\
\hline 3 & 0 & 28 & 86 \\
\hline 4 & 0 & 29 & 95 \\
\hline 5 & 0 & 30 & 104 \\
\hline 6 & 0 & 31 & 108 \\
\hline 7 & 0 & 32 & 120 \\
\hline 8 & 0 & 33 & 127 \\
\hline 9 & 0 & 34 & 128 \\
\hline 10 & 0 & 35 & 130 \\
\hline 11 & 12 & 36 & 130 \\
\hline 12 & 14 & 37 & 141 \\
\hline 13 & 19 & 38 & 142 \\
\hline 14 & 22 & 39 & 143 \\
\hline 15 & 23 & 40 & 143 \\
\hline 16 & 28 & 41 & 145 \\
\hline 17 & 37 & 42 & 147 \\
\hline 18 & 41 & 43 & 156 \\
\hline 19 & 56 & 44 & 156 \\
\hline 20 & 58 & 45 & 159 \\
\hline 21 & 58 & 46 & 163 \\
\hline 22 & 66 & 47 & 168 \\
\hline 23 & 66 & 48 & 179 \\
\hline 24 & 74 & 49 & 183 \\
\hline 25 & 78 & 50 & 185 \\
\hline
\end{tabular}

Table 3

Percent Correct Word Recognitions by Word Length and Word Frequency

\begin{tabular}{lcccc}
\hline & \multicolumn{4}{c}{ Word Length (N of Letters) } \\
\cline { 2 - 5 } \multicolumn{1}{c}{ Word Frequency* } & Four & Five & Six & Mean \\
\hline 1 Per Million & 29.66 & 24.34 & 25.40 & 26.47 \\
15 to 25 Per Million & 37.40 & 33.92 & 38.76 & 36.69 \\
100 to 400 Per Million & 44.86 & 44.26 & 44.00 & 44.37 \\
Mean & 37.31 & 34.17 & 36.05 & 35.84 \\
\hline
\end{tabular}

*Kučera and Francis, 1967 
nitions $(p>.40)$. The failure to find decreases in correct recognitions with increasing word length is unusual, and study of this anomolous finding is being pursued further.

\section{REFERENCES}

KuČera, H., \& Francis, W. N. Computational analysis of present-day American English. Providence, R.I: Brown University Press, 1967.

MAYZNER, M. S. Visual information processing of alphabetic inputs. Psychonomic Monograph Supplements, 1972, 4(13, Whole No. 6), 239-243.

MAYZNER, M. S. Studies of visual information processing in man. In R. L. Solso (Ed.), Information processing and cognition: The Loyola symposium. Hillsdale, N.J: Erlbaum, 1975. Pp. 31-54. (a)

MAYZNER, M. S. Visual information processing of letters and nonletters. Bulletin of the Psychonomic Society, 1975, 6, 421. (b)

MAYZner, M. S., \& Greenberg, J. Studies in the processing of sequentially presented inputs with overprinting paradigms. Psychonomic Monograph Supplements, 1971, 4(4, Whole No. 52), 73-84.

Mayzner, M. S., Tresselt, M. E., \& Helfer, M. S. A research strategy for studying certain effects of very fast sequential input rates on the visual system. Psychonomic Monograph Supplements, 1967, 2(5, Whole No. 21), 73-81.

(Received for publication December 7, 1977.) 